\title{
Favoriser la position d'apprentissage grâce à l'interaction superviseur-supervisé
}

\section{Fostering learning position through supervisor-trainee interaction}

\author{
Marie GIROUX et Gilles GIRARD \\ Département de médecine de famille, Faculté de médecine et des sciences de la santé Université de Sherbrooke $300112^{\mathrm{e}}$ \\ Avenue Nord, J1H 5N4 Sherbrooke, Québec, Canada
}

Manuscrit reçu le 26 février 2009; commentaires éditoriaux formulés aux auteurs le 13 juillet 2009; accepté pour publication le 15 septembre 2009

\begin{abstract}
Mots clés :
Position

d'apprentissage ;

stratégies de

supervision;

éducation médicale.

Résumé - Contexte et problématique : En supervision clinique, l' apprenant gagne à adopter certaines attitudes et cognitions lui permettant de mieux profiter des opportunités d'apprendre, constituant ainsi une «position d'apprentissage » c'est-à-dire orientée par l'apprentissage où l'évaluation est perçue surtout comme formative et intégrée au cheminement normal du médecin en devenir. Méthodes : Au fil des années, la mise à l'essai de divers exercices pratiques au cours d'ateliers a permis d'élargir la compréhension des conditions et stratégies concrètes pour y arriver, notamment la reconnaissance de la diversité des stratégies d'apprentissage et des obstacles susceptibles de s'y opposer. Résultats : L'intégration systématique d'un atelier sur le sujet dans le curriculum du programme d'études postdoctorales en médecine de famille et la référence fréquente à ce concept en supervision clinique constituent des retombées concrètes de ces travaux. Ces travaux de type recherche-action ont aussi permis de confirmer que le caractère ouvert et bienveillant des interactions entre le superviseur et le supervisé facilite l'adoption d'une position d'apprentissage, permettant à l'apprenant de prendre conscience de ses progrès graduels tout en renforçant son sentiment de compétence. Conclusion : La responsabilité de réunir les conditions facilitant une position orientée vers l'apprentissage incombe tant à l'apprenant qu'à son superviseur.
\end{abstract}

Keywords:

Learning position;

strategies of supervision; medical education.

Abstract - Context and problematic: When being supervised in clinical settings, learners benefit from adopting certain attitudes and cognitions allowing them to get the most out of learning opportunities. This constitutes a "learning position" in which evaluation is perceived primarily as formative and integrated into the normal physician training stream. Methods: Over the years, various practical exercises have been conducted during workshops, broadening knowledge about the elements that facilitate the learning position and about definite strategies for attaining it. Specific examples of this are learning strategies and potential impediments. Results: Integrating systematically a workshop on this topic into the curriculum for postgraduate training in family medicine and frequently referring to the concept during clinical supervision are concrete outcomes of this work. This action research also made it possible to confirm that the open, nurturing character of interactions between supervisor and supervisee facilitate the adoption of a learning position, allowing the learner to perceive his gradual progress and to bolster his feeling of competency. Conclusion: The learner and supervisor jointly share responsibility for bringing these facilitating conditions together. 


\section{Introduction}

Pendant la supervision clinique en médecine, il est souhaitable que l'apprenant adopte une position orientée par les apprentissages à effectuer que nous nommerons «position d'apprentissage » dans la suite du texte. Cette position se caractérise par une attitude d'ouverture, confiante, propice à expérimenter, à réfléchir et à tirer profit du partage de l'expérience disciplinaire et relationnelle des personnes ressources. Kilminster et ses collaborateurs $^{[1]}$ distinguent deux modalités de supervision : la supervision strictement clinique qui cible l'efficacité des pratiques médicales et la supervision pédagogique en contexte clinique qui, outre l'objectif précédent, vise à faciliter les apprentissages à effectuer par l'étudiant. La notion de position d'apprentissage en supervision s'inscrit avantageusement dans une perspective d'enseignement centré sur l'apprenant ${ }^{[2,3]}$, privilégiant la qualité de l'interaction superviseur-supervisé ${ }^{[1,4]}$. En éducation médicale, l'apprentissage et l'évaluation doivent susciter des activités convergentes, mutuellement contributives. Cependant, en supervision clinique, on constate que chaque apprenant adopte une position personnelle qui s'inscrit sur un continuum entre deux pôles à la fois complémentaires et opposés (schéma 1) : d'un côté, la "position d'apprentissage » et, de l'autre, la position guidée par les activités d'évaluation - que nous nommerons "position d'évaluation » dans la suite du texte. Cette dernière reflète davantage le fait d'être et de se sentir jugé et évalué. Elle est influencée notamment par la nature hiérarchique de la relation entre le superviseur et le supervisé, de même que par le désir de bien paraître chez l'apprenant. Un défi se pose donc : établir entre eux un climat de travail qui facilite la progression du supervisé vers la position d'apprentissage, qui favorise la perception de l'évaluation périodique et itérative comme étant surtout formative et intégrée au cheminement normal du médecin en devenir et qui soutient une exploitation optimale des opportunités d'apprentissage. Dans le présent article, les auteurs partagent leurs travaux et réflexions sur le sujet, qui se sont étalés sur une période de 15 ans.
Une démarche d'animation d'ateliers sur la position d'apprentissage auprès de résidents (internes, selon la terminologie europénne) et de superviseurs en médecine de famille, a été la pierre angulaire de ces travaux. À l'origine, ces ateliers visaient à convaincre les apprenants des bénéfices à attendre de l'adoption d'une position davantage orientée par les apprentissages ; il s'avéra que cet objectif ne pouvait pas être atteint sans que les superviseurs en soient eux-mêmes convaincus et participent activement à établir un dialogue pédagogique bienveillant et constructif avec les résidents. Étayée par une littérature portant sur des thèmes connexes, tels que le sentiment de compétence, la relation superviseursupervisé et les stratégies de supervision, cette démarche sera décrite avec ses résultats, ainsi que les retombées systémiques de ce cadre de référence innovateur. Des liens seront établis avec le rôle central d'une interaction superviseur-supervisé de qualité pour favoriser l'apprentissage pendant la formation clinique.

\section{Contexte et justification}

Au Canada, le programme de formation postdoctorale en médecine de famille menant à l'obtention d'un permis de pratiquer la médecine dure deux ans. Cette durée de formation s'avère controversée depuis de nombreuses années; plusieurs jugent que la somme des compétences à acquérir justifierait un programme plus long, d'autres estiment que deux ans suffisent à condition que les opportunités d'apprentissage soient investies de façon optimale. Le programme de l'université de Sherbrooke comporte un stage de six mois en unité de médecine de famille (UMF), au terme duquel les résidents doivent maitriser de nombreuses compétences essentielles à leur future pratique. De longue date, l'équipe professorale de ce programme, incluant les auteurs, se préoccupe activement de créer au cours du stage en UMF les conditions qui encouragent les résidents à profiter de façon optimale des opportunités d'apprentissage. 
Position d'évaluation (noir)

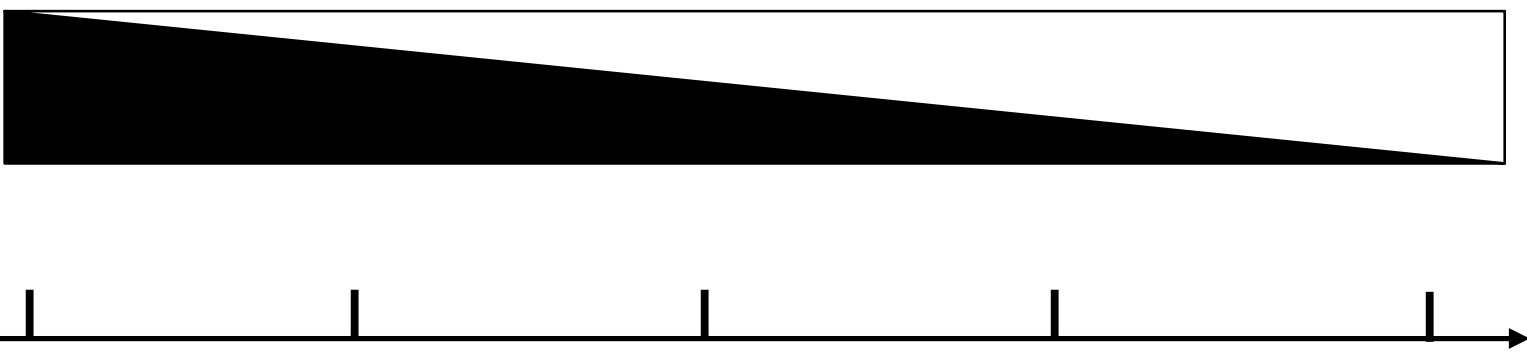

Schéma 1. Position d'évaluation versus position d'apprentissage.

Or, la culture médicale académique comporte ses rites de passage, dont certains ont un impact négatif sur les individus et les systèmes, par exemple l'utilisation encore trop fréquente du dénigrement ou de l'humiliation de l'apprenant par le superviseur, dans le but, avoué ou non, de stimuler sa motivation ou son apprentissage ${ }^{[5,6]}$. Au début des années 90, les auteurs ont fait l'hypothèse que des expériences antérieures de cette nature, ou d'autres croyances semblables erronées, pouvaient freiner l'apprentissage, en limitant l'attitude d'ouverture au cours des échanges en supervision clinique. Ils avaient l'intuition qu'une position d'apprentissage et une position d'évaluation, bien qu'elles témoignent chacune de leur réalité propre, peuvent entrer l'une l'autre en compétition. Quelques publications soutiennent cette thèse, parmi lesquelles une étude qualitative de Sommers et al. ${ }^{[7]}$ sur les croyances implicites d'étudiants de quatrième année en stage en médecine de famille, qui rapportent que ceux-ci perçoivent leurs premières supervisions par discussion de cas comme un test de compétence, au cours duquel ils devraient d'emblée se montrer compétents. À cet effet, ils cherchent à découvrir les attentes du superviseur et évitent les zones de doute ou d'incertitude en clinique, ce qui limite leur apprentissage. On y fait la démonstration que plus le superviseur exerce un contrôle directif de l'interaction à la manière d'un examinateur, plus le supervisé persiste dans la position d'évaluation.

De plus, au cours des dernières années, le milieu médical universitaire québécois s'est préoccupé de façon croissante de la prévention de l'intimidation

\section{Position d'apprentissage (blanc)}

et du harcèlement en milieu d'études et de travail ${ }^{[8]}$. Les signalements et plaintes à cet effet, de la part de résidents à l'endroit de superviseurs, sont régulièrement en lien avec le feedback et l'évaluation pendant les stages et des malentendus en sont souvent la source. L'environnement facultaire a été réceptif, de façon croissante, à des initiatives pédagogiques favorisant les échanges pédagogiques plus respectueux entre superviseurs et supervisés dans le but, aussi, de prévenir les perceptions d'intimidation ou de harcèlement.

\section{Méthodes}

Il y a plus de quinze ans, les auteurs se sont engagés dans une démarche itérative de type rechercheaction $^{[9]}$, entrecoupée de réflexions dans et après l'action. Ils ont d'abord développé un atelier de formation, de type «exploration avec visée de production » ${ }^{[10]}$ d'une durée de deux heures, s'adressant à des groupes de résidents de première année en médecine de famille en stage à l'UMF-Estrie, qui a évolué avec le temps en un atelier de type « exploration et apprentissage ${ }^{[10]}$. Le tableau I présente une comparaison du déroulement des ateliers dans leur forme initiale et dans leur forme actuelle.

Dès le début de cette démarche, l'objectif recherché auprès des résidents était de rendre explicite les éléments suivants : les différentes stratégies d'apprentissage chez l'adulte, les facteurs favorisant ou faisant obstacle à l'apprentissage en clinique, la différence entre position d'évaluation et position 
Tableau I. Évolution de l'atelier dédié à la « position d'apprentissage en supervision » au cours des 15 dernières années.

\begin{tabular}{|c|c|}
\hline $\begin{array}{l}\text { Plan de l'atelier initial } \\
\text { « type exploration avec visée de production » }\end{array}$ & $\begin{array}{c}\text { Plan de l'atelier actuel } \\
\text { « type exploration et apprentissage » }\end{array}$ \\
\hline $\begin{array}{l}\text { a. Introduction, objectifs et plan de l'atelier (5min) } \\
\text { b. Inventorier stratégies d'apprentissage par le biais } \\
\text { d'expérience personnelles : travail individuel, puis } \\
\text { présentation devant le groupe (25 min) } \\
\text { c. Définir les obstacles à l'apprentissage : discussion } \\
\text { en grand groupe (15 min) } \\
\text { d. Différencier position d'apprentissage et position } \\
\text { d'évaluation : exercice individuel écrit, mise en } \\
\text { commun et discussion ( } 25 \text { min) } \\
\text { e. Jeu de rôle : Favoriser la position d'apprentissage } \\
\text { chez le supervisé et le superviseur : exercice en } 2 \\
\text { temps devant le groupe (encadré } 1 \text { ) (25 min) } \\
\text { f. Étude de vignettes : Aider un résident à passer } \\
\text { d'une position d'évaluation à une position d'ap- } \\
\text { prentissage : travail en sous-groupes (encadré } 2 \text { ) * } \\
\text { (20 min) } \\
\text { g. Conclusion et évaluation de l'atelier (5 min) }\end{array}$ & $\begin{array}{l}\text { a. Introduction, objectifs et plan de l'atelier (5min) } \\
\text { b. Prioriser des stratégies personnelles d'apprentis- } \\
\text { sage favorisant la position d'apprentissage : travail } \\
\text { individuel à partir de contenus antérieurs (15 min) } \\
\text { c. Surmonter les obstacles à l'apprentissage : discus- } \\
\text { sion en grand groupe à partir des résultats anté- } \\
\text { rieurs (15 min) } \\
\text { d. Différencier position d'apprentissage et position } \\
\text { d'évaluation : comparaison des réponses sponta- } \\
\text { nées avec les contenus colligés auprès des groupes } \\
\text { antérieurs (20 min) } \\
\text { e. Jeu de rôle : Favoriser la position d'apprentissage } \\
\text { chez le supervisé et le superviseur : exercice en } 2 \\
\text { temps devant le groupe (encadré } 1 \text { ) (25 min) } \\
\text { f. Étude de vignettes : Aider un résident à passer } \\
\text { d'une position d'évaluation à une position d'ap- } \\
\text { prentissage : travail en sous-groupes (encadré } 2 \text { *) } \\
\text { (20 min) } \\
\text { g. Choisir de nouvelles stratégies de supervision favo- } \\
\text { risant la position d'apprentissage et discussion sur } \\
\text { les modalités d'application : travail individuel, dis- } \\
\text { cussion en grand groupe (15 min) } \\
\text { h. Conclusion et évaluation de l'atelier (5 min) } \\
\text { Total : } 120 \text { minutes }\end{array}$ \\
\hline
\end{tabular}

* Cet exercice est destiné aux ateliers s'adressant à des superviseurs.

d'apprentissage, les risques de la compétition potentielle entre les deux et les bénéfices d'adopter précocement dans leur formation toutes les attitudes, dispositions personnelles et comportements leur permettant de profiter au maximum de leur stage. Une partie importante de l'atelier était consacrée à un exercice interactif centré sur un jeu de rôle, invitant les résidents participants à expliciter la nature des échanges entre superviseur et supervisé, qui, pour reprendre la distinction terminologique évoquée précédemment, transformeraient une supervision strictement clinique en une supervision pédagogique en contexte clinique, visant à optimiser la position d'apprentissage du supervisé (encadré 1).

Lors de l'évaluation des premiers ateliers par les résidents participants, ces derniers se disaient enthousiastes et intéressés à exploiter ces «nouvelles » opportunités comme apprenants mais aussi inquiets que leurs superviseurs réels n'adhèrent pas aux mêmes valeurs; leur «nouvelle » ouverture à l'idée de davantage exprimer doutes et limites à leur superviseur pendant un stage risquait, selon eux, de se conclure par un revers de l'estime de soi ou, pire, une mauvaise évaluation! Une version enrichie 


Encadré 1
Jeu de rôle en deux temps (A et B) présenté devant le groupe
au cours de l'atelier dédié à la «position d'apprentissage en supervision »

\section{Jeu de rôle A}

1. Contexte du jeu de rôle : La scène du jeu de rôle comporte deux personnages, une résidente et son superviseur, joués par les animateurs de l'atelier, et se déroule à la fin d'une demi-journée de supervision par discussion de cas.

2. Consignes d'observation : La moitié du groupe observe le (la) résident(e) en vue de lui donner des suggestions pour améliorer sa position d'apprentissage et l'autre moitié du groupe observe le (la) superviseur(e) et prépare des suggestions pour bonifier le climat d'apprentissage.

3. Monologues du résident et du superviseur : Le superviseur, puis la résidente, exposent chacun leurs réflexions personnelles avant leur rencontre. Le superviseur ne dispose que de dix minutes pour conclure cette supervision, car il est attendu pour une réunion. Il se rassure en se disant que la résidente affiche de bonnes compétences pour son niveau et il envisage d'emblée une discussion de cas simple et courte. La résidente, pour sa part, sort exténuée de sa consultation. Le patient démontrait des signes persistants de colère en raison d'un retard du médecin. Il s'agit d'un jeune homme qui présente une lombalgie depuis une semaine, survenue au travail après avoir soulevé une lourde charge. De plus, la résidente est irritée des exigences reliées à cette consultation, ne se sentant pas pleinement compétente dans l'évaluation et le suivi des lombalgies. Sa séquence de l'examen clinique du rachis lombaire est désorganisée et ses repères anatomiques sont imprécis et lacunaires. Aussi réalise-t-elle après-coup qu'elle a oublié de rechercher le signe de Lasègue.

4. Scène de la supervision clinique : La résidente décrit le cas au superviseur de façon ordonnée et concise sans parler de ses malaises, de ses doutes et de son oubli, lequel, indiquerons-nous, n'avait pas d'incidence inquiétante dans le cas discuté compte tenu du reste de la cueillette de données. Le superviseur écoute sa présentation, constate qu'elle est globalement cohérente et donne son accord au diagnostic et à la conduite proposée, sans creuser davantage.

5. Feedback par les participants aux deux personnages : Chaque moitié du groupe est invitée à formuler ses commentaires et ses conseils au résident ou au superviseur afin que l'un et l'autre contribuent à améliorer le climat d'apprentissage.

\section{Jeu de rôle B}

1. Reprise de la scène de la supervision clinique, en version améliorée : Les acteurs intègrent les suggestions formulées par le groupe à la discussion de cas précédente.

2. Discussion en grand groupe : Les participants commentent cette nouvelle version de la supervision, en précisant si celle-ci leur apparaît ou non réaliste.

de l'atelier a donc aussi été offerte à des équipes mixtes incluant des superviseurs : celle-ci comportait un deuxième exercice interactif, réalisé à partir d'étude de vignettes, visant à outiller spécifiquement ces derniers pour aider les apprenants à se mobiliser davantage vers une position d'apprentissage (encadré 2).
Après chaque atelier, se déroulait un bilan entre animateurs avec révision des contenus évoqués et des évaluations fournies par les participants. Les données générées en groupe permettant de mieux comprendre la position d'apprentissage ont atteint un point de saturation après quelques mois. Dans les versions subséquentes de l'atelier, 


\section{\begin{tabular}{|c|}
\hline Encadré 2 \\
Étude de vignettes lors de l'atelier dédié à la « position d'apprentissage en supervision »
\end{tabular}}

\section{Consignes :}

1. Division du groupe des participants en sous-groupes de cinq à six individus

2. Travail en sous-groupes : Lecture d'une des deux vignettes et réponse aux questions

3. Retour en groupe pour une mise en commun des réponses, avec commentaires des animateurs

Vignette \#1 Sylvie

Sylvie est en stage à l'UMF (unité de médecine de famille) depuis 1 mois. Elle fonctionne bien et est efficace. Ses histoires de cas sont bien ficelées et sans anicroche, ses hypothèses diagnostiques claires, ses notes concises et elle cumule peu de retard pour une résidente de première année. D'ailleurs, elle vous a dit ne pas aimer être en retard. Lors de ses deux supervisions directes, elle a rencontré des patients peu compliqués qui généraient chez elle peu de questionnements. Suite à deux anecdotes où le superviseur a trouvé des nouvelles données pertinentes en retournant questionner le patient, vous avez émis l'hypothèse qu'elle ne va peut-être pas toujours au fond des choses.

Vignette \#2 Marianne

Marianne est en stage à l'UMF depuis trois mois. Plus le stage avance, plus on accumule des exemples et des anecdotes qui témoignent clairement de lacunes importantes au niveau des connaissances et du raisonnement clinique. Dès le début du stage, son équipe de superviseurs avait identifié certaines lacunes et a effectué un soutien et un encadrement plus serré mais les différentes stratégies pédagogiques adoptées, avec ses efforts, ne lui ont pas permis d'atteindre ses objectifs. Lors de la première évaluation formative, vous avez évoqué avec elle la possibilité qu'elle ne valide pas son stage. Depuis ce temps, vous sentez que Marianne est sur la défensive : elle minimise ses difficultés, affiche souvent une confiance en elle inadéquate et affirme qu'elle a encore trois mois pour s'améliorer. Elle a dit à un des superviseurs que vous avez des préjugés à son égard et que la supervision plus serrée est devenue pour elle une source de stress importante qui explique sa contreperformance.

1. Où situez-vous ces deux résidentes sur le continuum «position d'évaluation versus position d'apprentissage »?

\begin{tabular}{ccccc} 
Position d'évaluation & & \multicolumn{2}{c}{ Position d'apprentissage } \\
$\mathbf{1}$ & $\mathbf{2}$ & $\mathbf{3}$ & $\mathbf{5}$ \\
$\mathrm{I}$ & $\mathrm{I}$ & $\mathrm{I}$ & $\mathrm{I}$
\end{tabular}

1. Pouvons-nous les aider à se mobiliser davantage vers une position d'apprentissage?

Si oui, comment? 
les productions des groupes antérieurs étaient présentées et discutées avec les participants, permettant davantage d'efficience et de profondeur dans les discussions en groupe. L'atelier dans sa forme « exploration et apprentissage » abordait un contenu plus riche, notamment la composante relationnelle du climat d'apprentissage et ce, sans en modifier la durée. Ceux-ci ont été offerts en moyenne trois à quatre fois par année et dans divers contextes : lors des activités d'enseignement formel aux résidents en stage en UMF du programme de l'université de Sherbrooke, lors des journées départementales annuelles de formation professorale et lors de congrès nationaux et internationaux en pédagogie médicale.

\section{Résultats}

Définitions enrichies des positions d'évaluation et d'apprentissage

Au fil des années, les participants ont contribué à mieux caractériser ces deux concepts et ce, sous différents angles, à partir de leurs expériences respectives comme apprenant et comme superviseur (tableau II). En résumé, un apprenant en position d'évaluation aspire surtout à ce que sa performance soit perçue comme adéquate et conforme aux attentes du superviseur, en vue de transmettre une image extérieure de compétence. Il a tendance à montrer ce qu'il sait faire et à taire ses points à améliorer ressentis comme des faiblesses. L'apprenant en position d'apprentissage s'avère plus confiant et centré sur la progression dans ses apprentissages et les moyens pour y accéder. Il est à l'affût des opportunités d'apprendre, expérimente davantage et expose ouvertement ses forces et ses points à améliorer, en vue d'être guidé dans ses apprentissages. Bref, il s'approprie davantage la finalité de sa formation médicale.

Stratégies d'apprentissage chez l'adulte et impacts sur la position d'apprentissage

Dans la forme initiale des ateliers, les participants ont identifié plusieurs stratégies d'apprentissage différentes chez l'adulte, parmi lesquelles les auteurs ont distingué cinq catégories (tableau III) dont les quatre premières sont décrites dans l'étude qualitative de Teunissen et al. ${ }^{[11]}$ portant sur la description du processus d'apprentissage de résidents en gynéco-obstétrique à l'aide de groupes de discussion. Le tableau III dresse la liste de ces stratégies ou fournit des exemples concrets pour chacune d'elles. Après saturation des données, une discussion de groupe a été intégrée à cet exercice, portant sur les impacts de différentes stratégies sur l'adoption d'une position d'apprentissage. Les participants soulignent que les stratégies 3,4 et 5, bien que moins fréquemment rapportées, facilitent le passage d'une position d'évaluation à une position d'apprentissage. L'apprenant qui recourt à des références théoriques (stratégie 1) est d'emblée moins porté à expérimenter mais plus intéressé à se documenter pour améliorer sa performance. Celui qui privilégie la méthode par essais et erreurs (stratégie 2) expérimente facilement mais faute, de repères fiables, risque de se décourager rapidement en cas d'échecs répétés. Toutefois, l'apprenant qui a bénéficié antérieurement d'une bonne expérience de supervision par un entraîneur (coaching), par exemple dans la pratique d'un sport ou d'une activité artistique (stratégie 3), démontre plus de facilité à adopter une position d'apprentissage. Stimulé par les apports reçus de l'entourage, il a aussi souvent développé de bonnes habiletés d'analyse réflexive (stratégie 4) et a appris à s'efforcer d'adopter les attitudes et conditions facilitant l'apprentissage (stratégie 5). La perception d'une progression personnelle tangible constitue ce qui motive le plus l'adoption et le maintien d'une position d'apprentissage.

Variables ayant une incidence sur l'apprentissage et interventions sur mesure par le superviseur

L'apprentissage étant un processus dynamique et complexe, le superviseur gagne à tenir compte de différentes variables afin de faciliter la transition de l'apprenant d'une position d'évaluation vers une position d'apprentissage. Les participants à l'atelier évoquent plusieurs interventions du superviseur 
Tableau II. Définitions enrichies des positions d'évaluation et d'apprentissage.

\begin{tabular}{|c|c|c|}
\hline & Position d'évaluation & Position d'apprentissage \\
\hline $\begin{array}{c}\text { Besoins } \\
\text { et motivations } \\
(\mathrm{BM})\end{array}$ & $\begin{array}{l}\text { - Paraître compétent. } \\
\text { - Être conforme aux attentes. } \\
\text { - Sentir l'approbation. } \\
\text { - Obtenir une évaluation « agréable ». }\end{array}$ & $\begin{array}{l}\text { - Devenir compétent. } \\
\text { - Apprendre et expérimenter. } \\
\text { - Puiser sa motivation dans la perception de } \\
\text { progresser dans son apprentissage. } \\
\text { - Obtenir une évaluation qui lui donne l'heure } \\
\text { juste. }\end{array}$ \\
\hline Pensées (P) & $\begin{array}{l}\text { - Présente de la rigidité. } \\
\text { - Affiche une vision à court terme, axée sur } \\
\text { ce qui est sûr. } \\
\text { - Ignore les éléments discordants dans } \\
\text { la démarche clinique et contourne } \\
\text { ses lacunes. } \\
\text { - Tente de combler les zones grises par ses } \\
\text { connaissances préexistantes. } \\
\text { - Révèle certaines distorsions dans son } \\
\text { auto-évaluation. } \\
\text { - Vise à fournir « la bonne réponse », quitte } \\
\text { à « deviner » les attentes du superviseur. } \\
\text { - Visualise de façon prédominante d'obtenir } \\
\text { de bonnes évaluations et de passer avec } \\
\text { succès les examens. }\end{array}$ & $\begin{array}{l}\text { - Présente de la souplesse. } \\
\text { - Affiche une vision à moyen et long terme, } \\
\text { - Reconnaît ses compétences à bonifier } \\
\text { et participe de façon consensuelle à un plan } \\
\text { pédagogique le concernant. } \\
\text { - Veut élargir ses horizons et intègre plusieurs } \\
\text { points de vue. } \\
\text { - Considère les zones grises comme } \\
\text { des opportunités d'apprentissage. } \\
\text { - Fait preuve d'authenticité dans } \\
\text { son autoévaluation. } \\
\text { - Considère plusieurs hypothèses et tolère } \\
\text { les zones grises. } \\
\text { - Se visualise en pratique et s'inspire de la } \\
\text { finalité de ses compétences. }\end{array}$ \\
\hline Émotions (É) & $\begin{array}{l}\text { - Présente un sentiment de fragilité } \\
\text { par rapport à sa compétence. } \\
\text { - Présente un stress interne de performance } \\
\text { susceptible de freiner son développement. } \\
\text { - Ressent ses lacunes comme des faiblesses, } \\
\text { consciemment ou non. } \\
\text { - Vit le statut de débutant avec une certaine } \\
\text { honte. }\end{array}$ & $\begin{array}{l}\text { - Éprouve un sentiment de confiance face } \\
\text { aux tâches proposées. } \\
\text { - Se sent moins stressé et interagit plus } \\
\text { spontanément. } \\
\text { - Normalise le statut de débutant, qui est vécu } \\
\text { de façon sereine. } \\
\text { - Souhaite volontiers faire confiance } \\
\text { au superviseur quant au fait que ce dernier } \\
\text { n'abusera pas de son pouvoir. }\end{array}$ \\
\hline
\end{tabular}


Tableau II. Suite.

\begin{tabular}{|c|c|c|}
\hline & Position d'évaluation & Position d'apprentissage \\
\hline $\begin{array}{l}\text { Comportements } \\
\text { (C) }\end{array}$ & $\begin{array}{l}\text { - Expose ce qu'il sait faire. } \\
\text { - Tait ou camoufle ses points à améliorer. } \\
\text { - Choisit les cas faciles qu'il maîtrise } \\
\text { et évite les cas difficiles. } \\
\text { - Raconte ses cas comme si tout était } \\
\text { conforme à son diagnostic. } \\
\text { - Fait des choix « stéréotypés » d'objectifs } \\
\text { d'apprentissage personnel. }\end{array}$ & $\begin{array}{l}\text { - Montre à la fois ses forces et ses points } \\
\text { à améliorer. } \\
\text { - Choisit les cas difficiles qui représentent } \\
\text { pour lui un défi pour apprendre. } \\
\text { - Présente ses cas en énonçant ses questions, } \\
\text { malaises et lacunes. } \\
\text { - Nomme ses véritables objectifs personnels } \\
\text { d'apprentissage. }\end{array}$ \\
\hline $\begin{array}{l}\text { Attitudes face } \\
\text { à l'apprentissage } \\
\text { et au feedback } \\
\text { (A) }\end{array}$ & $\begin{array}{l}\text { - Affiche une attitude fermée et défensive } \\
\text { avec une peur du risque. } \\
\text { - Se justifie face à la critique perçue } \\
\text { comme un jugement négatif sur } \\
\text { sa compétence et même une menace à } \\
\text { son intégrité. } \\
\text { - Nie les difficultés rencontrées. } \\
\text { - Minimise les commentaires défavorables } \\
\text { contenus dans son évaluation. }\end{array}$ & $\begin{array}{l}\text { - Témoigne d'une volonté de progresser et } \\
\text { d'une ouverture à prendre les risques } \\
\text { appropriés pour y arriver. } \\
\text { - Accepte la critique perçue d'emblée comme } \\
\text { constructive et nécessaire à l'apprentissage. } \\
\text { - Aborde spontanément les difficultés } \\
\text { rencontrées. } \\
\text { - Questionne sur les commentaires } \\
\text { défavorables contenus dans son évaluation } \\
\text { afin de mieux en saisir la portée. }\end{array}$ \\
\hline $\begin{array}{c}\text { Relation avec } \\
\text { le superviseur } \\
\text { (Rel) }\end{array}$ & $\begin{array}{l}\text { - Établit une relation dominant-dominé où } \\
\text { il se sent évalué et jugé. } \\
\text { - Adhère au point de vue du superviseur, } \\
\text { car veut être perçu comme « correct» } \\
\text { et afficher la bonne réponse. }\end{array}$ & $\begin{array}{l}\text { - Établit une relation d'égal à égal où les } \\
\text { deux partenaires apprennent. } \\
\text { - Pose des questions, génère des discussions } \\
\text { et se prononce personnellement. } \\
\text { - Peut faire preuve de prudence dans son } \\
\text { ouverture avec un superviseur } \\
\text { potentiellement intimidant. }\end{array}$ \\
\hline $\begin{array}{l}\text { Autocritique } \\
\text { (AC) }\end{array}$ & $\begin{array}{l}\text { - Se surévalue ou se sous-évalue et } \\
\text { se montre réticent à nuancer son } \\
\text { évaluation. } \\
\text { - Éprouve de la difficulté à faire le lien } \\
\text { entre la rétroaction verbale quotidienne } \\
\text { et l'évaluation sommative. }\end{array}$ & $\begin{array}{l}\text { - Manifeste une bonne capacité d'auto- } \\
\text { évaluation réaliste (analyse réflexive). } \\
\text { - Intègre naturellement la rétroaction } \\
\text { quotidienne dans sa compréhension de } \\
\text { son évaluation sommative. }\end{array}$ \\
\hline
\end{tabular}


Tableau III. Impacts des stratégies d'apprentissage sur la position d'apprentissage.

\begin{tabular}{|c|c|c|}
\hline $\begin{array}{l}\text { Catégories de stratégies } \\
\text { d'apprentissage (par ordre } \\
\text { de fréquence d'évocation) }\end{array}$ & Exemples & $\begin{array}{c}\text { Impacts sur la position } \\
\text { d'apprentissage } \\
\text { (rang accordé par participants) }\end{array}$ \\
\hline $\begin{array}{l}\text { 1. Recours à des références } \\
\text { théoriques. }\end{array}$ & $\begin{array}{l}\text { - Suivre un cours magistral. } \\
\text { - Utiliser des ouvrages de référence. } \\
\text { l'apécomposer cognitivement } \\
\text { progressives. }\end{array}$ & $\begin{array}{l}5^{e} \text { rang } \\
\text { - Aide à percevoir les exigences } \\
\text { de la tâche. }\end{array}$ \\
\hline $\begin{array}{l}\text { 2. Exploration individuelle } \\
\text { de type essai-erreur. }\end{array}$ & $\begin{array}{l}\text { - Expérimenter à son rythme. } \\
\text { - Apprendre par essai-erreur. } \\
\text { - Pratiquer sur le terrain et de façon } \\
\text { répétée. }\end{array}$ & $\begin{array}{l}4^{e} \text { rang } \\
\text { - Indique une ouverture à } \\
\text { l'expérimentation. } \\
\text { - N'implique pas la présence de } \\
\text { témoin. }\end{array}$ \\
\hline $\begin{array}{l}\text { 3. Discussion et échanges } \\
\text { avec d'autres. }\end{array}$ & $\begin{array}{l}\text { - Observer des modèles. } \\
\text { - Prendre un cours pratique. } \\
\text { - Obtenir du feedback. }\end{array}$ & $\begin{array}{l}3^{e} \text { rang } \\
\text { - Suppose une ouverture au feedback. }\end{array}$ \\
\hline $\begin{array}{l}\text { 4. Analyse réflexive de sa } \\
\text { performance avec autocor- } \\
\text { rection. }\end{array}$ & $\begin{array}{l}\text { - Se regarder dans l'activité } \\
\text { (ex. : utilisation du vidéo). } \\
\text { - Se visualiser dans ce qu'on } \\
\text { apprend. } \\
\text { - S'auto-corriger. } \\
\text { - Planifier une progression dans } \\
\text { l'apprentissage. }\end{array}$ & $\begin{array}{l}2^{e} \text { rang } \\
\text { - Témoigne d'un intérêt à progresser } \\
\text { et d'habiletés de métacognition }{ }^{[11]} \\
\text { (autorégulation des apprentissages). }\end{array}$ \\
\hline $\begin{array}{l}\text { 5. Adoption d'attitudes per- } \\
\text { sonnelles d'ouverture facili- } \\
\text { tant l'apprentissage et dis- } \\
\text { cussion de ses préoccupa- } \\
\text { tions quant aux fluctuations } \\
\text { normales de son sentiment } \\
\text { de compétence }{ }^{[12]} \text {. }\end{array}$ & $\begin{array}{l}\text { - Adopter un statut de « débutant». } \\
\text { - Accepter la phase initiale } \\
\text { de déstabilisation ou de remise } \\
\text { fonctionnement. } \\
\text { - Doser les expériences d'échecs } \\
\text { et de succès. } \\
\text { - Tenir compte de ses goûts. } \\
\text { - Créer un contexte de plaisir. }\end{array}$ & $\begin{array}{l}1^{\text {er }} \text { rang } \\
\text { - Met en évidence une ouverture à } \\
\text { apprendre et une flexibilité à adapter } \\
\text { ses attitudes en conséquence. }\end{array}$ \\
\hline
\end{tabular}


Tableau IV. Variables ayant une incidence sur l'apprentissage et interventions sur mesure par le superviseur.

\begin{tabular}{|c|c|}
\hline Variables & Interventions sur mesure \\
\hline $\begin{array}{l}\text { A) Type de contenu } \\
\text { - connaissances déclaratives (le quoi) : } \\
\text { - connaissances procédurales (le comment) : } \\
\text { - connaissances conditionnelles (le pourquoi et le } \\
\text { quand) : }\end{array}$ & $\begin{array}{l}\text { - Proposition de tâches d'élaboration et d'organi- } \\
\text { sation (ex. : construction de schémas). } \\
\text { - Action (ex. : démonstration et pratique de sé- } \\
\text { quences d'action avec options). } \\
\text { - Discrimination (ex. : utilisation de contre- } \\
\text { exemples) et généralisation (ex. : utilisation des } \\
\text { «si»). }\end{array}$ \\
\hline $\begin{array}{l}\text { B) Niveau de l'apprenant } \\
\text { - novice : passe peu de temps à analyser les don- } \\
\text { nées du problème et consacre la majorité de son } \\
\text { temps à tenter des solutions; } \\
\text { - expert : accorde de l'importance et du temps à } \\
\text { l'analyse des données du problème et des impacts } \\
\text { des solutions. }\end{array}$ & $\begin{array}{l}\text { - Anticipation guidée (ex. : "pré-coaching » en } \\
\text { supervision : préciser avec le résident son plan } \\
\text { d'action avant de voir le patient et, suite à la } \\
\text { consultation, discuter des ajustements de son } \\
\text { plan et des raisons qui les ont motivés). }\end{array}$ \\
\hline $\begin{array}{l}\text { C) Style cognitif de l'apprenant } \\
\text { (façon personnelle d'aborder la réalité sur les plans } \\
\text { intellectuel et affectif) } \\
\text { - réaliste : axé sur les faits, } \\
\text { - pragmatiste : axé sur ce qui fonctionne, } \\
\text { - idéaliste : axé sur les personnes et les valeurs, } \\
\text { - analyste : axé sur la logique et la structure, } \\
\text { - synthétiste : axé sur l'intégration de diverses } \\
\text { perspectives. }\end{array}$ & $\begin{array}{l}\text { - Prise en compte du style de son interlocuteur et } \\
\text { de son propre style. } \\
\text { - Reconnaissance que chacun présente des affini- } \\
\text { tés naturelles avec ceux qui partagent les mêmes } \\
\text { styles qu'eux et peut éprouver des difficultés à in- } \\
\text { teragir avec un interlocuteur affichant des styles } \\
\text { qu'il a peu développés. }\end{array}$ \\
\hline $\begin{array}{l}\text { D) Motivation de l'apprenant } \\
\text { - perception de ses capacités à accomplir la tâche } \\
\text { avec succès : sentiment de compétence ou d'effi- } \\
\text { cacité personnelle }^{[16]} \text {, } \\
\text { - perception élevée de la valeur de la tâche, } \\
\text { - perception d'une certaine autonomie et d'un } \\
\text { contrôle sur son apprentissage. }\end{array}$ & $\begin{array}{l}\text { - Facilitation d'expériences personnelles de succès } \\
\text { face à des tâches présentant un défi intéressant. } \\
\text { - Discussion avec l'apprenant pour l'aider à uti- } \\
\text { liser positivement ses expériences d'échec qui } \\
\text { risquent de mettre en cause son sentiment de } \\
\text { compétence et de réduire ses efforts à la tâche. }\end{array}$ \\
\hline
\end{tabular}


reconnues comme efficaces dans la littérature en lien avec ces variables : le type de contenu ${ }^{[13]}$ à apprendre, le niveau de l'apprenant ${ }^{[13]}$, le style cognitif $^{[14]}$ de l'apprenant et du superviseur et surtout la motivation ${ }^{[15]}$ de l'apprenant (tableau IV). Ainsi, pour motiver un apprenant novice qui présente un style pragmatiste et des difficultés à acquérir certaines connaissances procédurales, on aura avantage à faciliter les expériences de succès et à limiter la répétition d'expériences d'échecs par la démonstration initiale des procédures en cause, l'anticipation guidée de son plan d'action et la pratique des séquences d'action avec feedback et soutien sur mesure par rapport aux étapes qui lui posent un défi.

Reconnaissance des obstacles à la position d'apprentissage

Dans la version initiale des ateliers, les participants identifiaient les obstacles fréquents à la position d'apprentissage. On y reconnaît trois grandes catégories : les limites personnelles physiques ou psychologiques, les conjonctures défavorables sur le plan organisationnel et les cognitions anxiogènes reliées au stress de performance chez l'apprenant ou son superviseur. Parmi les situations-pièges, la fatigue, souvent inhérente à la vie de médecin en apprentissage ou en pratique, de même que les impératifs cliniques et le manque de temps, expliquent le manque de dialogue stimulant l'apprentissage entre le superviseur et l'apprenant. Parmi les croyances erronées et autres distorsions cognitives, le monologue intérieur honteux de l'apprenant («Je suis supposé savoir cela. » et «Il ne faut pas montrer ce que l'on ne sait pas. »), renforce la position d'évaluation et limite les opportunités de dialogue. Les participants soulignent l'importance de reconnaitre ces obstacles au quotidien et de les nuancer pour favoriser et maintenir une position d'apprentissage. Dans cette partie de l'atelier, les échanges entre animateurs et participants permettent aussi d'éviter que le discours ne tombe dans un dogmatisme pédagogique irréaliste. On normalise certaines des situations-piège évoquées qui font partie de la réalité clinique et culturelle de la collectivité médicale universitaire.

\section{Genèse de stratégies favorisant la position d'apprentissage}

Les éléments favorisant la position d'apprentissage sont de plusieurs ordres : les exercices interactifs ont mis en évidence plusieurs exemples concrets de ces conditions gagnantes, dont certaines sont sous la responsabilité du résident $(\mathrm{R})$ et d'autres, de son superviseur (S). En somme, les auteurs ont constaté que ces conditions ont trait aux catégories de caractéristiques qui décrivent la position d'apprentissage : besoins / motivations (BM), pensées (P), émotions (É), attitudes (A), comportements $(\mathrm{C})$, relation avec l'autre (Rel), autocritique (AC) (tableau 2). Ces codes seront utilisés afin de classifier les interventions proposées par les participants.

\section{Premier exercice}

Le premier exercice se réfère à une situation classique de supervision clinique. Les participants constatent que plusieurs supervisions se déroulent ainsi et que cela s'avère une "norme » acceptable puisque, somme toute, le cas a été géré de façon efficace et sans risque pour le patient. Dans le but de susciter un climat favorisant davantage la position d'apprentissage, ils génèrent à l'intention du superviseur, puis de la résidente, plusieurs suggestions simples, qui initient entre eux un dialogue plus authentique. Ils indiquent au superviseur qu'il aurait avantage à faire part à la résidente de sa contrainte de temps (S-BM) mais qu'il a le souci de bien superviser celle-ci (S-É), quitte à compléter sa supervision plus tard au besoin (S-C). Afin d'assurer une supervision pédagogique ${ }^{[1,4]}$ à la résidente et de profiter de l'opportunité d'apprentissage, les participants suggèrent au superviseur de lui demander de formuler une question soulevée par sa consultation avant même de présenter le cas (S-C). Une telle 
proposition vise à rendre explicite le fait que le superviseur dépasse la finalité exclusivement soignante de l'évaluation, comme cela est le cas dans une supervision strictement clinique ${ }^{[1,4]}(\mathrm{S}-\mathrm{P})$. La résidente, elle, est invitée à faire part au superviseur du climat inconfortable de la consultation qu'elle n'a pas réussi à désamorcer (R-P), de ses lacunes dans l'évaluation des lombalgies (R-C) et de son oubli de faire la manœuvre de Lasègue (R-A), ce dont elle n'est pas fière (R-É). Les participants soulignent auprès des deux personnages que leurs échanges manquaient de cohérence avec leur réalité respective $(\mathrm{R}$ et $\mathrm{S}-\mathrm{AC}$ ) et les invitent à faire preuve entre eux de plus d'ouverture et d'authenticité ( $\mathrm{R}$ et $\mathrm{S}-\mathrm{Rel}$ ), qui sont deux caractéristiques essentielles d'une position orientée vers l'apprentissage.

Le jeu de rôle est ensuite rejoué en une version améliorée qui intègre les suggestions des participants et le tout ne dure que sept minutes au lieu de cinq. Le superviseur démontre brièvement à la résidente les repères anatomiques de l'examen du rachis lombosacré (S-C). Il lui propose une référence pertinente susceptible de l'aider à peaufiner sa séquence d'examen du dos $(\mathrm{S}-\mathrm{C})$ et s'engage à la vérifier avec elle lors du prochain rendez-vous du patient (S-BM). Il l'invite également à valider brièvement la colère du patient avec ce dernier en vue de la désamorcer (S-C). La résidente, qui reconnaît l'importance de bien savoir évaluer les lombalgies dans le cadre de sa future pratique (R-BM), exprime au superviseur comment elle s'est sentie irritée par ce cas (R-É) qui lui posait un défi clinique et relationnel avec le patient : parmi ses zones de doute (R-A), elle choisit une question pertinente à approfondir avec son superviseur en lien avec ce cas-ci (R-C) et s'engage elle aussi à rediscuter prochainement des lombalgies avec son superviseur (R-Rel). Le caractère réaliste du jeu de rôle en version améliorée, même avec la contrainte de temps connue, constitue un moment fort, crédible et convaincant de l'atelier. On y fait aussi la démonstration sans équivoque que favoriser la position apprentissage se joue à deux, notamment par un échange ouvert et bienveillant entre l'apprenant et son superviseur.

\section{Deuxième exercice}

Le deuxième exercice interactif, réalisé à partir d'études de vignettes, permet aux participants de situer les deux résidentes en position d'évaluation (à une cote de 1 ou 2 sur l'échelle proposée) mais chacune des vignettes témoigne d'une réalité différente. Dans la vignette \#1, Sylvie présente un bon rendement global mais évite les opportunités de questionnements personnels sur sa performance (R-C et A), ce qui l'amène à négliger des données pertinentes avec les patients (R-P). Les participants suggèrent de rappeler à la résidente que nos interventions visent à favoriser ses apprentissages (S-BM). Ainsi, en lui reflétant nos observations et nos préoccupations (S-P), on pourrait l'inciter à faire preuve de plus d'ouverture (S-C). En outre, en l'encourageant par l'évocation de ses réussites (S-Rel), on pourrait l'aider à approfondir sa démarche clinique en acceptant de se questionner et de corriger ses lacunes (S-A). Cela sera possible en créant avec elle une relation de confiance (S-Rel) où elle se sentirait rassurée par rapport à ses compétences de base (R-É) et confiante pour accepter de nouveaux objectifs d'apprentissage (R-A). Turgeon et al. ${ }^{[17]}$ rapportent l'expérience documentée d'une résidente en supervision directe qui, après quelques mois, a transformé un souci initial de bien paraître face au superviseur en un réel désir d'apprendre grâce à une relation superviseursupervisé sécurisante où elle s'est sentie confirmée dans son identité professionnelle. Ceci s'est traduit, entre autres, par la décision d'exposer en supervision les problèmes posés par ses patients jugés plus difficiles.

Dans la vignette \#2, les participants concluent que la position d'évaluation adoptée par Marianne se comprend aisément, car elle risque de faire face aux conséquences réelles d'un échec en raison d'une progression insuffisante malgré un encadrement pédagogique adéquat. Les participants proposent de la responsabiliser par rapport à un risque accru d'échec (S-BM) si elle persiste dans son refus de coopérer à l'encadrement proposé. Cette tâche sera difficile si sa méfiance envers ses superviseurs persiste (R-Rel) et l'empêche de percevoir la pertinence 
d'un encadrement pédagogique étroit ( $\mathrm{R}-\mathrm{A}$ et $\mathrm{P}$; $\mathrm{S}-\mathrm{C})$ pour l'aider à résoudre ses difficultés $(\mathrm{R}-\mathrm{C})$. Cette vignette permet au groupe d'aborder l'incontournable caractère sanctionnel de l'évaluation, de souligner l'importance que cette dernière soit bien documentée et, même en présence de difficultés pédagogiques significatives, de reconnaître la plus value de privilégier des interactions fermes, mais respectueuses, avec l'apprenant (S-C).

\section{Discussion}

Intégration à la culture pédagogique en supervision

À l'origine de cette démarche, la position d'apprentissage avait été abordée comme étant surtout sous la responsabilité de l'apprenant. L'apport des participants a mis en évidence qu'il était incontournable de parler aussi des stratégies de supervision pédagogique du superviseur et de la relation entre le superviseur et l'apprenant.

Le vocabulaire et le cadre conceptuel entourant la position d'apprentissage ont été intégrés progressivement à la culture pédagogique de notre département de médecine de famille. Les stratégies sur mesure pour aider nos apprenants à y parvenir sont intégrées aux discussions dans les réunions d'équipe de superviseurs et dans les échanges superviseurrésident. Ceci a été facilité par la participation active des nouveaux groupes d'enseignants et de résidents aux rééditions annuelles de l'atelier. Chez les apprenants présentant des difficultés importantes, l'adoption d'une position d'apprentissage est perçue par les superviseurs comme un facteur de meilleur pronostic puisqu'elle témoigne d'une meilleure autocritique et d'une meilleure identification des lacunes à combler. Chez ceux dont les performances cliniques sont déjà satisfaisantes, la position d'apprentissage prend la forme de démarches métacognitives stimulantes pour les apprenants et les professeurs. Plusieurs résidents qui ont participé à l'atelier sur la position d'apprentissage mentionnent qu'ils auraient eu avantage à être sensibilisés plus tôt à cette formation, c'est-à-dire au niveau de l'externat. Toutefois, ils apprécient également que les superviseurs et les résidents d'un même milieu le suivent conjointement. Des stratégies de supervision ont été identifiées et priorisées par les enseignants et résidents de notre département spécifiquement pour cultiver un climat de travail favorisant la position d'apprentissage, perçue comme un pré-requis pour optimiser le sentiment de compétence des résidents.

\section{Arrimages avec le curriculum}

Lors de la dernière révision de notre curriculum en 2001-2003 ${ }^{[18]}$, les enseignants et résidents du programme ont été consultés de façon itérative pour, entre autres, améliorer la supervision clinique au quotidien et optimiser le sentiment de compétence du résident de même que la position d'apprentissage. Lors d'ateliers et de groupes de discussion focalisée (focus groups) entre pairs, deux cohortes de 70 enseignants et résidents ont formulé, puis validé, 69 stratégies de supervision dont 20 ont été priorisées (annexe 1). Les 20 stratégies proposées visent à développer le sentiment de compétence du résident et à optimiser la position d'apprentissage. Elles mettent l'accent sur les dimensions suivantes : la stimulation des questions chez le résident et des temps réservés pour y répondre, le processus d'autoévaluation et de réflexion du résident, la discussion ouverte à propos de l'évolution et des fluctuations normales du sentiment de compétence ${ }^{[12]}$, la clarification des attentes du superviseur, les ingrédients d'un feedback constructif comme soutien à l'auto-évaluation ${ }^{[19]}$, le soutien et l'encouragement à l'autonomie et l'alternance des rôles d'observé et d'observateur témoignant de l'impact du modèle de rôle ${ }^{[20]}$ en supervision. Le comité de révision du programme a recommandé que l'atelier sur la position d'apprentissage fasse partie intégrante du curriculum de base pour tous les nouveaux résidents, qui sont répartis dans sept UMF différentes. Les auteurs ont intégré à l'atelier un exercice portant spécifiquement sur ces 20 stratégies les plus populaires et les 
enseignants de ces différents milieux de stage y ont participé en grand nombre lors de journées de formation professorale.

\section{Liens avec un climat d'apprentissage plus sain}

Puisque des interactions respectueuses et ouvertes entre le superviseur et le supervisé sont essentielles au climat favorisant l'apprentissage, une des suites logiques de ces travaux a consisté dans l'élaboration et l'adoption à l'intérieur de notre faculté de médecine d'un code de conduite du superviseur dans sa relation avec le supervisé ${ }^{[21]}$. La prévention du harcèlement psychologique ayant été identifiée comme prioritaire dans plusieurs milieux universitaires académiques, des conférences et ateliers sont régulièrement offerts dans notre milieu aux résidents et aux enseignants, au cours desquels l'invitation à favoriser la position d'apprentissage est réitérée à tous. Les étudiants et résidents en médecine, qui consultent des adjoints à la vie étudiante en raison de stress relié au statut de débutant, apprécient le recadrage apporté par le concept de position d'apprentissage, qui normalise cette étape de leur formation médicale.

Retombées et limites de l'étude

L'évolution fertile de ces travaux, de même que l'intérêt et la mobilisation qu'ils ont suscités au sein des groupes d'apprenants et de superviseurs sur une période de 15 ans, témoignent de la pertinence du propos. Dans leur évaluation des ateliers, les participants nous ont souvent confié qu'ils voyaient dans la notion de position d'apprentissage, des indices encourageants de l'évolution de la culture en éducation médicale ; lorsque l'humiliation, le dénigrement et la peur y prédominent, les superviseurs et apprenants réalisent avec déception que nos comportements intra-professionnels ne sont pas à la hauteur des valeurs prônées en professionnalisme médical et dans la relation médecin-patient. Des changements sociodémographiques et générationnels chez les apprenants, de même que l'évolution des connaissances en pédagogie médicale, nous amènent à considérer la culture médicale comme l'un des déterminants de l'efficacité de l'apprentissage. Ces travaux, dont l'ampleur éventuelle avait été sous-estimée au départ, n'ont toutefois pas fait encore l'objet de mesures d'impacts en ce qui concerne les stratégies facilitant le passage d'une position d'évaluation à une position d'apprentissage.

\section{Conclusion}

Initiée dans le but de promouvoir des conditions personnelles gagnantes chez l'apprenant en vue d'optimiser ses apprentissages, cette démarche d'animation d'atelier sur la position d'apprentissage a connu des retombées surprenantes et fécondes avec le temps, dont une influence positive et constructive sur le climat de travail et d'apprentissage dans nos unités d'enseignement. Elle a permis de caractériser plus rigoureusement les positions d'apprentissage et d'évaluation et de faire des liens explicites avec l'application concrète de stratégies d'apprentissage en supervision clinique. De plus, elle a constitué un lieu de parole légitime pour aborder sous l'angle pédagogique la plus value d'une culture médicale plus saine, notamment en soulignant l'importance de la qualité des interactions et échanges entre superviseur et supervisé. L'idée de susciter et de cultiver la position d'apprentissage en supervision clinique constitue un cadre de référence fertile, innovateur et mobilisateur, en accord avec les valeurs émergentes en pédagogie médicale.

\section{Contributions}

Les auteurs ont contribué collégialement et solidairement à la conception et à la rédaction de l'article. 


\begin{tabular}{|c|c|}
\hline \multicolumn{2}{|c|}{$\begin{array}{l}\text { Annexe } 1 \\
\text { avorisant l'adoption d'une position d'apprentissage } \\
\text { sentiment de compétence du résident }\end{array}$} \\
\hline Formulations du résident (R) & Formulations du superviseur (S) \\
\hline $\begin{array}{l}\text { 1. Identifier périodiquement avec mon } S \text { où j'en } \\
\text { suis dans ma formation, partager mes inquiétudes } \\
\text { et préciser mes buts et mes attentes d'encadrement, } \\
\text { car c'est ma formation. }\end{array}$ & $\begin{array}{l}\text { 1. Identifier périodiquement avec le } \mathrm{R} \text { où il en est dans } \\
\text { sa formation en l'amenant à partager ses inquiétudes } \\
\text { et à préciser ses buts et ses attentes d'encadrement, car } \\
\text { c'est sa formation. }\end{array}$ \\
\hline $\begin{array}{l}\text { 2. Demander à mon } \mathbf{S} \text { de verbaliser ses attentes par } \\
\text { rapport à ma performance tenant compte de mon niveau } \\
\text { de formation; cela peut être bien différent des percep- } \\
\text { tions que j'ai de ses attentes. }\end{array}$ & $\begin{array}{l}\text { 2. En tant que } S \text {, énoncer au résident mes attentes en } \\
\text { termes de performance attendue tenant compte de ses } \\
\text { propres attentes et des objectifs de son niveau de forma- } \\
\text { tion. }\end{array}$ \\
\hline $\begin{array}{l}\text { 3. Profiter des cas discutés pour énoncer au } S \text { une ou } \\
\text { deux questions que je me pose et y répondre conjoin- } \\
\text { tement. }\end{array}$ & $\begin{array}{l}\text { 3. Profiter des cas supervisés pour inciter le } \mathrm{R} \text { à énoncer } \\
\text { une ou deux questions qu'il se pose et à y répondre } \\
\text { avec notre aide. }\end{array}$ \\
\hline $\begin{array}{l}\text { 4. Négocier avec le } S \text { des temps protégés réalistes pré- } \\
\text { cis pour discuter de questions à approfondir. }\end{array}$ & $\begin{array}{l}\text { 4. Réserver des temps protégés réalistes précis pour } \\
\text { discuter d'une question à approfondir. }\end{array}$ \\
\hline $\begin{array}{l}\text { 5. Identifier à quel point je me sens compétent (e) } \\
\text { dans les diverses activités de mon stage et discuter avec } \\
\text { mon } S \text { du prochain pas à franchir pour me sentir plus }\end{array}$ & $\begin{array}{l}\text { 5. Vérifier auprès du résident son sentiment de compé- } \\
\text { tence dans les diverses activités de son stage et discuter } \\
\text { de ce qui pourrait l'aider à se sentir plus compétent. }\end{array}$ \\
\hline $\begin{array}{l}\text { compétent(e). } \\
\text { 6. Désactiver les pressions que je m'impose sur le plan } \\
\text { des connaissances à acquérir et des tâches à accomplir et, } \\
\text { au besoin, discuter avec mon } \mathrm{S} \text { de ce qui est important à }\end{array}$ & $\begin{array}{l}\text { 6. Désactiver les pressions que s'impose le } \mathrm{R} \text { au niveau } \\
\text { des connaissances à acquérir et des tâches à accomplir, } \\
\text { en partageant notre propre vécu de } \mathrm{R} \text { et en soulignant } \\
\text { l'important à son niveau de formation. }\end{array}$ \\
\hline $\begin{array}{l}\text { mon niveau de formation. } \\
\text { 7. Avant de demander un feedback, procéder d'abord à } \\
\text { une auto-évaluation de mon travail et la communiquer }\end{array}$ & $\begin{array}{l}\text { 7. Avant de livrer un feedback, inciter d'abord le R à pro- } \\
\text { céder à l'auto-évaluation de son travail, puis la faire } \\
\text { nuancer à l'aide de questions. }\end{array}$ \\
\hline $\begin{array}{l}\text { au } \mathrm{S} \text {. } \\
\text { 8. Me faire confiance ! Me féliciter de mes bons coups ! } \\
\text { Les valider avec mon } \mathrm{S} \text {. }\end{array}$ & $\begin{array}{l}\text { 8. Souligner explicitement les bons coups du } \mathrm{R} \text { et ses } \\
\text { points à améliorer de manière à ce qu'il ne perçoive pas } \\
\text { uniquement ces derniers. }\end{array}$ \\
\hline $\begin{array}{l}\text { 9. En fin de supervision, discuter du point à améliorer } \\
\text { en terme d'objectif à travailler lors de la prochaine su- } \\
\text { pervision. }\end{array}$ & $\begin{array}{l}\text { 9. En fin de séance de supervision, transformer le point } \\
\text { à améliorer en terme d'objectif à travailler lors de la } \\
\text { prochaine supervision. }\end{array}$ \\
\hline $\begin{array}{l}\text { 10. Entendre les points positifs, pas seulement les points } \\
\text { négatifs lors des supervisions et discuter en quoi le feed- } \\
\text { back reçu modifie mon auto-évaluation. }\end{array}$ & $\begin{array}{l}\text { 10. Suite au feedback, vérifier ce que le } \mathrm{R} \text { entend au sujet } \\
\text { de ses points forts et de ses points à améliorer et en quoi } \\
\text { le } \text { feedback reçu modifie son auto-évaluation. }\end{array}$ \\
\hline $\begin{array}{l}\text { 11. Lorsqu'une interaction s'annonce difficile avec un } \\
\text { patient, convenir avec mon } \mathbf{S} \text { avant l'entrevue d'un } \\
\text { objectif ou d'un plan d'intervention (pré-coaching) et } \\
\text { discuter des ajustements effectués en cours d'entrevue. }\end{array}$ & $\begin{array}{l}\text { 11. Faire de la supervision anticipatoire (pré- } \\
\text { coaching) en révisant avec le R son plan d'action ou des } \\
\text { points importants à considérer avant qu'il rencontre le } \\
\text { patient et discuter par la suite des ajustements effectués } \\
\text { en entrevue. }\end{array}$ \\
\hline
\end{tabular}




\begin{tabular}{|l|}
\hline \multicolumn{1}{|c|}{ Formulations du résident $(\mathrm{R})$} \\
\hline 12. Suite à une consultation serrée dans le temps où j'ai \\
éprouvé un sentiment d'incompétence avec un patient \\
difficile, revoir ce que je suis parvenu(e) à couvrir \\
lors de cette rencontre.
\end{tabular}

13. Demander au S de l'observer en situations d'entrevue avec des patients difficiles de manière à percevoir mes compétences et les faire ressortir au moment d'une discussion après l'entrevue.

14. Lorsque le $\mathrm{S}$ et le $\mathrm{R}$ voient conjointement les patients (ex. : tournées à l'hôpital), alterner les rôles d'observateur et d'intervenant avec retour critique sur les entretiens et discussion des questions litigieuses.

15. Utiliser le vidéo pour m'observer en entrevue et le visionner avec mon $S$ pour identifier mes forces et des façons d'améliorer ma performance.

16. Présenter mon cas au $\mathrm{S}$ avec un souci de synthèse comme si je transférais le dossier à un collègue. Au besoin, résumer le cas par écrit en spécifiant les questions que je me pose.

17. Apprendre l'efficacité dans la gestion de mon temps : discuter avec le $\mathrm{S}$ de la façon dont $\mathrm{j}$ 'aurais pu gagner du temps avec tel patient, comment j'aurais pu couper dans mon questionnaire ou mon examen.

18. Me voir comme le «médecin traitant » du patient et considérer le $\mathrm{S}$ comme un «coach» et un collègue de travail plutôt que simplement comme un évaluateur.

19. Lorsque RII, utiliser la technique des deux piles de dossiers : une première pile à réviser rapidement et une seconde à revoir à partir de questions que je me pose sur les cas.

20. Donner un feedback au $S$ par rapport à l'activité de supervision en regard de mes propres attentes.

Synthèse des énoncés : Élaine Blanchard, résidente, et Gilles Girard.
Formulations du superviseur (S)

12. Suite à une consultation serrée dans le temps où le $\mathrm{R}$ a éprouvé un sentiment d'incompétence avec un patient difficile, revoir avec lui ce qu'il a réussi à accomplir lors de cette rencontre.

13. Permettre au R d'observer comment nous nous débrouillons dans les situations difficiles de manière à ce qu'il perçoive ses compétences et les faire ressortir dans une discussion après l'entrevue.

14. Lorsque le $\mathrm{R}$ et le $\mathrm{S}$ voient conjointement les patients (ex. : tournées à l'hôpital), alterner les rôles d'observateur et d'intervenant avec retour critique sur les entretiens et discussion des questions litigieuses.

15. Utiliser le vidéo pour que le $\mathrm{R}$ observe ses forces, qu'il voit comment s'améliorer et qu'il prenne une saine distance par rapport à sa performance.

16. Pour aider le R à percevoir ses compétences de synthèse, lui demander de résumer son cas et ses questions par écrit avant d'en discuter comme s'il transférait le dossier à un collègue.

17. Enseigner l'efficacité dans la gestion du temps : montrer au $\mathrm{R}$ comment il aurait pu gagner du temps avec tel patient, comment il aurait pu couper dans son questionnaire ou son examen.

18. Proposer au RII de vivre les prochaines semaines comme s'il était médecin autonome et de me voir comme un coach ou un collègue de travail avec qui il échange pour améliorer sa performance.

19. Avec le RII, utiliser la technique des deux piles de dossiers : une première pile à réviser rapidement et une seconde à revoir à partir des questions du $\mathrm{R}$.

20. Demander au $\mathrm{R}$ du feedback par rapport à ma supervision.

RII : Résident de deuxième année

\section{Références}

1. Kilminster S, Cottrell D, Grant J, Jolly B. AMEE Guide No 27 : Effective educationaland clinical supervision. Med Teach 2007;29:2-19.

2. Cate O, Snell L, Mann K, Vermunt J. Orienting Teaching Toward the Learning Process. Acad Med 2004;79:21928.
3. Jouquan J, Bail P. À quoi s'engage-t-on en basculant du paradigme d'enseignement vers le paradigme d'apprentissage ? Pédagogie Médicale 2003;4: 163-75.

4. Martineau B, Girard G, Boulé R. Interventions en supervision directe pour développer la compétence du résident : une recherche qualitative. Pédagogie Médicale 2008;9:19-31.

5. Frank E, Carrera JS, Stratton T, Bickel JN, Lois M. Experiences of belittlement and harassment and their 
correlates among medical students in the United States: longitudinal survey. BMJ 2006;333:682-7.

6. Mareiniss DP. Decreasing GME Training Stress to Foster Residents' Professionalism. Acad Med 2004;79:825-31.

7. Sommers PS, Muller JH, Saba GW, Draisin JA, Shore WB. Reflections-on-action: Medical Students' Accounts of their Implicit Beliefs and Strategies in the Context of One-to-One Clinical Teaching. Acad Med 1994;69(suppl. 10):S84-7.

8. Collège Royal des médecins et chirurgiens du Canada, Collège des médecins de famille du Canada, Collège des médecins du Québec. L'agrément et la question de l'intimidation et du harcèlement dans la formation médicale postdoctorale : lignes directrices pour les visiteurs et les programmes, 2004. [On- line]. Disponible sur : http://www.cmq. org/EtudiantsResidents/Agrement/ /media/ 848E72E89FED40A5B9E673F6CE2D9866. ashx? 90923

9. St-Arnaud Y. Connaître par l'action. Montréal: PUM, 1992.

10. Girard G, Clavet D, Boulé R. Planification et animation de trois types d'ateliers pédagogiques. Pédagogie Médicale 2008;9:42-53.

11. Teunissen PW, Scheele F, Scherpbier AJJA, Van Der Vleuten CPM, Boor K, Van Luijk SJ, Van DiemenSteenvoorde JAAM. How residents learn: qualitative evidence for the pivotal role of clinical activities. Med Educ 2007;41:763-70.

12. Speechley M, Weston WW, Dickie GL, Orr V. Selfassessed competence before and after residency. Can Fam Phys 1994;40:459-64.

13. Tardif J. Pour un enseignement stratégique. L'apport de la psychologie cognitive. Montréal: Éditions Logiques, 1992.
14. Harrison AE, Bramson RM. Styles of thinking: Strategies for asking questions, making decisions, and solving problems. New York: Anchor Press Doubleday, 1982.

15. Pelaccia T, Delplancq H, Triby E, Léman C, Bartier JC, Dupeyron JP. La motivation en formation: une dimension réhabilitée dans un environnement d'apprentissage en mutation. Pédagogie Médicale 2008 9:103-21.

16. Bandura A. Self-efficacy: Toward a Unifying Theory of Behavioural Change. Psych Rev 1977;84:191-215.

17. Turgeon J, St-Hilaire S. La supervision directe en médecine familiale, l'expérience d'une résidente. Pédagogie Médicale 2001;2:199-205.

18. Hatcher S, Clavet D. Nouvelles Orientations du Programme de Résidence en Médecine de Famille, Faculté de Médecine et des sciences de la santé, Université de Sherbrooke, september 2004.

19. Westberg J, Jason H. Fostering Reflection and Providing Feedback. New York : Springer Publishing Company, 2001.

20. Chamberland M, Hivon R. Les compétences de l'enseignant clinicien et le modèle de rôle en formation clinique. Pédagogie Médicale 2005;6:98-111.

21. Giroux M, Bergeron D. Un code de conduite du superviseur dans sa relation avec le supervisé lors de la formation clinique en médecine. Pédagogie Médicale 2003;4:202-7.

Correspondance et offprints : Marie GIROUX, Département de médecine de famille, Faculté de médecine et des sciences de la santé Université de Sherbrooke $300112^{\mathrm{e}}$ Avenue Nord, J1H 5N4 Sherbrooke, Québec, Canada.

Mailto : Marie.Giroux@USherbrooke.ca 